\title{
Short communication \\ Stability and phase separation behaviour of systems of particles in a medium with added polymer
}

\author{
Habauka M Kwaambwa \\ Chemistry Department, University of Botswana, Private Bag 00704, Gaborone, BOTSWANA
}

\begin{abstract}
The stability of barium carbonate $\left(\mathrm{BaCO}_{3}\right)$ suspensions in the presence of the dispersants, xanthan gum, sodium carboxymethylcellulose and carrageenan, has been studied using a phase separation technique. These materials have been found to stabilise the $\mathrm{BaCO}_{3}$ suspensions by increasing the viscosity of the medium. Of the three polymer dispersants, xanthan gum is the most effective in stabilising and destabilising the suspensions. The experimental results are compared to the phase separation and stability of non-aqueous latex suspensions in the presence of non-adsorbing (free) polymers. The systems consist of poly (hydroxystearic) acid (PHS) coated poly(methylmethacrylate) (PMMA) particles in dodecane and well-characterized cispoly(isoprene) (PIP) and poly(isobutylene) as the non-adsorbing added polymers.
\end{abstract}

Keywords: critical polymer concentration; depletion flocculation; dispersant; non-adsorbing polymer; phase separation; sedimentation; stability

\section{Introduction}

The interaction between colloidal particles is of paramount importance in industrial solid-liquid separation processes and is therefore one of the main topics in colloid science. In polar media, such as water, the interaction between colloids is often well described by the DLVO theory, which combines Van der Waals attraction between particles and repulsion due to overlap of the ion clouds surrounding the particles (Verwey and Overbeek, 1948). Although this theory successfully explains the stability of many colloidal systems, the existence of so-called non-DLVO forces plays a crucial role in a number of industrial colloidal formulations and processes. Colloids can also be sterically stabilized by chemically or physically attaching polymers to the particle surface (Israelachvili, 1992; Tadros, 1982). In good solvents or theta solvents for the polymer, colloids repel each other when overlap of their polymer layers forces the polymers into entropically unfavourable conformations.

It is well-known that the addition of soluble, non-adsorbing (free) polymer to a colloidal dispersion modifies the inter-particle interactions and hence the stability characteristics (Tadros, 1982). While the polymer as an adsorbed species may have a stabilising effect on the dispersion, the free polymer in solution has been shown to have the opposite effect in the semi-dilute regime. As the polymer concentration in the continuous phase increases, a critical polymer concentration is reached above which the free polymer is excluded from the inter-particle space. The exclusion of the polymer due to depletion or loss of configurational entropy results in attractive force, which is proportional to the osmotic pressure of the polymer in the bulk solution, between the particles. This attractive force results in particle dispersion instability referred to as depletion flocculation. The phenomenon

+267 3555039; fax: +2673552836

e-mail: kwaambwa@mopipi.ub.bw

Received 14 April 2005; accepted in revised form 10 August 2005. of depletion flocculation is relevant to prevention of formation of dilatant sediments upon sedimentation.

The manufacture of building materials such as bricks, tiles and ceramics nearly always involves water-soluble of sulphates of calcium, magnesium, potassium and sodium. Portland cements, clay and sand used in the mortar and grout during construction are usually the sources of the water-soluble salts. In the case of brick manufacture, these sulphates may cause crystallisation and efflorescence on bricks (Loewenthal et al, 1986). In the efflorescence, many test programmes have been developed and numerous attempts have been made to eliminate the problems caused by water-soluble salts. Unfortunately, nothing has proven $100 \%$ effective against this stubborn problem.

Barium carbonate $\left(\mathrm{BaCO}_{3}\right)$ is a light coloured powder used as a chemical additive in brick- and glass-making. It is known to be effective at eliminating sulphates by reacting to form a precipitate, which can easily be removed (Trusler et al, 1991). In other words, $\mathrm{BaCO}_{3}$ immobilizes the sulphates and renders them insoluble. This prevents the salts from being dissolved into solution that could migrate through the wall to the surface. Fine particles of carbonate are more effective, so it is usually applied in powder form with particle sizes ranging from 0.25 to $2 \mu \mathrm{m}$. However, this powder poses a significant health hazard and is also difficult to preserve and handle. A suspension of barium carbonate would be easier and safer to use; however, previously tested formulations have been unstable, settling or destabilising if left for a week or more.

In this paper, the effects of adding different dispersants in order to form a stable formulation of $\mathrm{BaCO}_{3}$ suspension are considered. Dispersants are useful in preventing settling, deposition, precipitation, agglomeration or caking of solid particles in a fluid medium (Nussinovitch, 1997). Biopolymeric dispersants used in this study were xanthan gum, sodium carboxymethylcellulose (CMC) and carrageenan. Adding small amounts of the dispersant to an aqueous suspension can significantly increase its stability even in changing temperatures and after many months. Such stable suspensions could also prove useful for removing water-soluble sulphates in effluent treatment 
and electrolytic processes (Friend and Loewenthal, 1992; Poon, 1973). The experimental results are compared to the phase separation and stability of non-aqueous latex suspensions in the presence of non-adsorbing (free) polymers. The systems for comparison consist of poly(hydroxystearic) acid (PHS) coated poly(methylmethacrylate) (PMMA) particles in dodecane and cis-poly(isoprene) (PIP) and poly(isobutylene) as the nonadsorbing added polymers.

\section{Experimental}

\section{Preparation, stability and phase separation of $\mathrm{BaCO}_{3}$ aqueous dispersions}

$\mathrm{BaCO}_{3}$ (density, $\rho=4.43 \mathrm{~g} \cdot \mathrm{cm}^{-3}$ ), xanthan gum (practical grade) and CMC (weight-average molecular mass, $M_{w}=250000$ ) were obtained from Sigma-Aldrich and used without further purification. Carrageenans were extracted using alkaline aqueous solution from marine algae Iridea harvested from the central shore of Namibia near Walvis Bay town (Nussinovitch, 1997; Dickinson, 1992; Mshingeni and Semesis, 1977).

To prepare the suspensions, $10 \mathrm{~g} \mathrm{BaCO}_{3}$ was weighed into a stoppered glass bottle and $190 \mathrm{~g}$ distilled water was added to give a $5 \% \mathrm{w} / \mathrm{w} \mathrm{BaCO}_{3}$ suspension. The suspension was stirred magnetically for $1 \mathrm{~h}$ after which $2 \mathrm{~g}$ of the resulting dispersion were weighed into glass vials followed by varying amounts of polymer. One vial was used as a control in which no polymer was added. The mixtures were shaken vigorously in order to dissolve the polymer additive. The samples were left to stand at room temperature for several days ranging from 5 to $10 \mathrm{~d}$ during which time the data for the ratio of sediment height $\left(H_{S}\right)$ to total dispersion height $\left(H_{T}\right)$, called fractional boundary ratio, were recorded as a function of polymer concentration. It was generally observed that the interface between the solvent-rich phase and the particle-rich phase was well-defined within a week and so the $H_{S} / H_{T}$ data reported here were made on the fifth day. The ratio $H_{S} / H_{T}=1$ implies a stable dispersion at the time of recording the measurement.

\section{Preparation, stability and phase separation of PMMA non-aqueous dispersions model systems}

Poly(methylmethacrylate) (PMMA) latex dispersions sterically stabilised with chemically grafted poly(12-hydroxy-stearic acid) (PHS) chains, and dispersed in dodecane were prepared using the method described by Antl et al. (1986). The PHS chains are terminally grafted at multiple points along the backbone of an insoluble anchor polymer PMMA.

The particle size was determined by transmission electron microscopy (TEM) for particle core size and photon correlation spectroscopy (PCS) for hydrodynamic size. The adsorbed layer thickness derived from TEM and PCS compared well with those obtained using the capillary viscometry method based on Saunders and the Krieger-Dougherty viscosity-volume fraction relationship (Goodwin and Hughes, 2000). Table 1 shows the particle core radius, $a$, obtained from TEM and the mean adsorbed layer thickness, $\delta$, from different methods for each of the lattices prepared (Kwaambwa, 1995; Reynolds and Reid, 1991).

The additive polymers namely cis-poly (isoprene) (PIP) and poly(isobutylene) (PIB), were obtained from Polymer Laboratory Ltd. and used without treatment. The polymers were supplied with commercial molecular mass characterisation data such as weight-average molecular mass, $M_{w}$, number-average molecular mass, $M_{n}$, viscosity-average molecular mass, $M_{v}$, and poly-dispersity, $M_{w} / M_{n}$. The polymers had low poly-dispersity $\left(M_{w} / M_{n} \leq 1.05\right)$. Table 2 shows some molecular mass data including intrinsic viscosities, [ $\eta$ ], and radius of gyration, $R_{g}$. For a given polymer molecular mass, the relative solution viscosity of the polymer in the solvent at $289 \mathrm{~K}$ was measured using capillary viscometry. This was performed at several polymer concentrations. These data were extrapolated linearly by Huggins and Kramer plots to find the intrinsic viscosities at infinite dilution. The $R_{g}$ values of the polymers were derived from the measured intrinsic viscosities (Goodwin et al., 2000 and 2004; Van Kravelen, 1976).

The stability and phase separation behaviour of the PMMA dispersions was studied in the presence of these added nonadsorbing polymers, PIP and PIB. A stock dispersion of latex of known volume fraction and high PIP or PIB concentration was prepared. The samples of varying polymer concentration were then prepared in similar screw-top, glass vials by diluting down with stock latex dispersion. The required amounts of stock dispersion and polymer were added gravimetrically (weighing the vial after each addition). The concentration of the additive polymer was expressed in $\mathrm{g}^{\cdot} \mathrm{cm}^{-3}$ in the continuous phase. The samples were allowed to stand at room temperature. The data for the ratio $H_{S} / H_{T}$ as function of polymer concentration was recorded over a period of time (at least two weeks for most sample series).

\begin{tabular}{|l|c|c|}
\hline \multicolumn{3}{|c|}{ TABLE 1 } \\
\begin{tabular}{|l} 
Particle sizes of the lattices \\
(a= particle core radius, $\delta=$ \\
adsorbed layer thickness)
\end{tabular} \\
\hline $\begin{array}{l}\text { Latex } \\
\text { code }\end{array}$ & $\begin{array}{c}\text { Radius, } \\
\text { a } / \mathbf{n m}\end{array}$ & $\delta / \mathbf{n m}$ \\
\hline HK5 & 180 & 3.5 \\
HK8 & 180 & 9 \\
\hline
\end{tabular}

\begin{tabular}{|l|r|r|c|c|c|}
\hline \multicolumn{7}{|c|}{$\begin{array}{c}\text { TABLE } 2 \\
\text { Molecular mass and radius of gyration of PIP } \\
\text { polymers used }\end{array}$} \\
\hline $\begin{array}{l}\text { Polymer } \\
\text { code }\end{array}$ & $\mathbf{M}_{\mathbf{w}}$ & $\mathbf{M}_{\mathrm{v}}$ & $\mathbf{M}_{\mathbf{w}} / \mathbf{M}_{\mathbf{n}}$ & $\begin{array}{c}{[\eta] /} \\
\mathbf{c m}^{3} \mathbf{g}^{-1}\end{array}$ & $\mathbf{R}_{\mathbf{g}} / \mathbf{n m}$ \\
\hline P31K & 31500 & 31257 & 1.03 & 28.23 & 7.7 \\
P115K & 115000 & 110000 & 1.02 & 68.37 & 17.4 \\
PIB & 380000 & 362000 & 1.05 & ---- & 32 \\
\hline
\end{tabular}

\section{Results and discussions}

\section{Phase separation of $\mathrm{BaCO}_{3}$ dispersions with added polymer}

Figure 1 shows a plot of the fractional boundary ratio, $H_{S} / H_{T}$ as a function of polymer concentration. Progressive addition of polymer resulted in an initial decrease (phase separation) in the ratio followed by a gradual increase and then a stable region. Phase separation occurred faster for $\mathrm{BaCO}_{3}$ suspensions without added polymer than systems with added polymer and the solvent-rich phase was clearer. It was not possible to determine the critical polymer concentration, $c^{+}$, taken in this paper as the added polymer concentration in the continuous phase at which dispersions start to phase separate. It was, however, possible to estimate the critical polymer concentration at which the ratio $H_{S} / H_{T}$ starts to go up and this was taken to be the critical polymer concentration at which restabilisation begins, $c^{++}$. The $c^{++}$values per 20 $\mathrm{g} \mathrm{BaCO}_{3}$ were estimated to be $0.007 \mathrm{~g}, 0.010 \mathrm{~g}$ and $0.015 \mathrm{~g}$ for xanthan gum, CMC and carrageenan, respectively. 


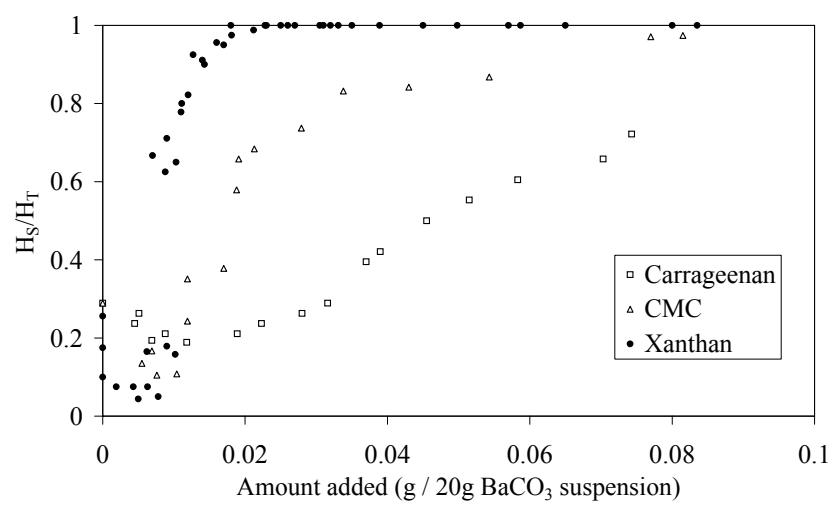

Figure 1

Phase separation of $\mathrm{BaCO}_{3}$ suspensions with different added additives

The results show that phase separation was not solely due to depletion flocculation but also as a result of density differences between the particles and solvent and the viscosity of the medium. For instance, the equilibrium velocity, $V_{o}$, at which a spherical particle of radius $a$ and density $\rho_{\mathrm{p}}$ will fall in a liquid of density $\rho_{\mathrm{m}}$ and viscosity $\eta_{\mathrm{m}}$ is given by Stokes law, expressed by the equation:

$$
\text { where: } \quad V_{o}=\frac{2 a\left(\rho_{p}-\rho_{m}\right) g}{9 \eta_{m}}
$$

$g$ is the acceleration due to gravity.

The above treatment applies to very dilute suspensions of noninteracting particles. According to Stokes law, the sedimentation rate decreases with decreasing difference in specific gravity of medium and particles and increasing medium viscosity. A balance of the density of disperse phase and medium retards settling. In this particular case the density difference between $\mathrm{BaCO}_{3}$ and water was very pronounced, so that systems without added polymer had a faster sedimentation rate. As the polymer concentration increases, the density difference of the particles and medium decreases and therefore, it becomes more and more difficult for the particles to settle. Increased viscosity will slow down the sedimentation but (unless the continuous phase possesses a yield value i.e. is a weak gel, which traps the solid particles) the sedimentation rate will never reach zero. The initial decrease in the ratio $H_{S} / H_{T}$ may be an indication of instability or flocculation of particles caused by bridging and/or depletion when the polymer is added.

Figure 1 also compares the effectiveness of the three dispersants in stabilising $\mathrm{BaCO}_{3}$ suspensions. Clearly, xanthan gum is the most effective dispersant (stabilizer) followed by CMC and carrageenan. It is also the most effective destabiliser since the ratio $H_{S} / H_{T}$ is smallest at $c^{++}$polymer concentration. The anionic polyelectrolyte xanthan is an especially efficient thickener because it strongly increases the continuous phase viscosity at lower concentrations. This is due to its high molar mass (order of $10^{6} \mathrm{~g} / \mathrm{mol}$ ) and semi-rigid molecular structure (persistence length $120 \mathrm{~nm}$ ) (Koenderink et al., 2003) as compared to carrageenan whose molar mass and persistence length is about $2-3.5 \times 10^{6}$ $\mathrm{g} / \mathrm{mol}$ and $50 \mathrm{~nm}$, respectively (Nishini and Takahashi, 2003).

\section{Phase separation of PMMA dispersions with added polymer}

The phase separation behaviour of PMMA dispersions with added polymers PIP $\left(M_{w}=31500,115000\right)$ and PIB $\left(M_{w}=\right.$

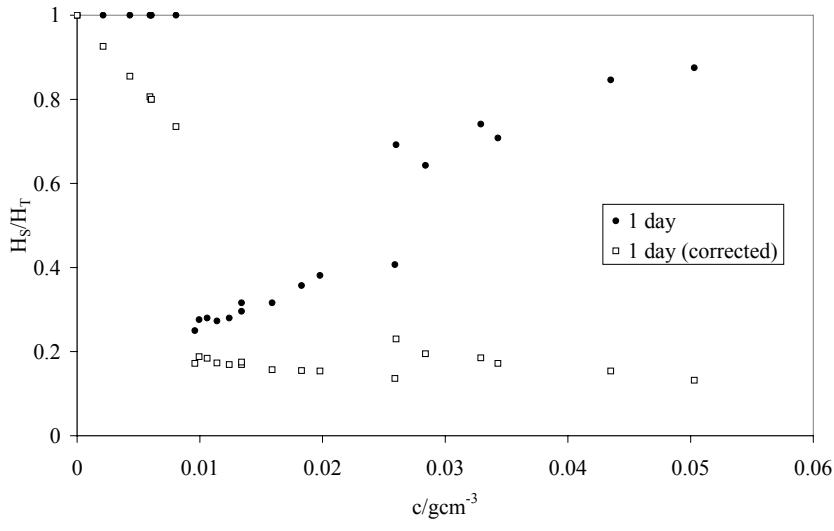

Figure 2

Typical phase separation results of PMMA with added PIP (HK5 $\left.a=180 \mathrm{~nm}, \delta=3.5 \mathrm{~nm}, \phi=0.100 \mathrm{M}_{w}(P I P)=115000\right)$

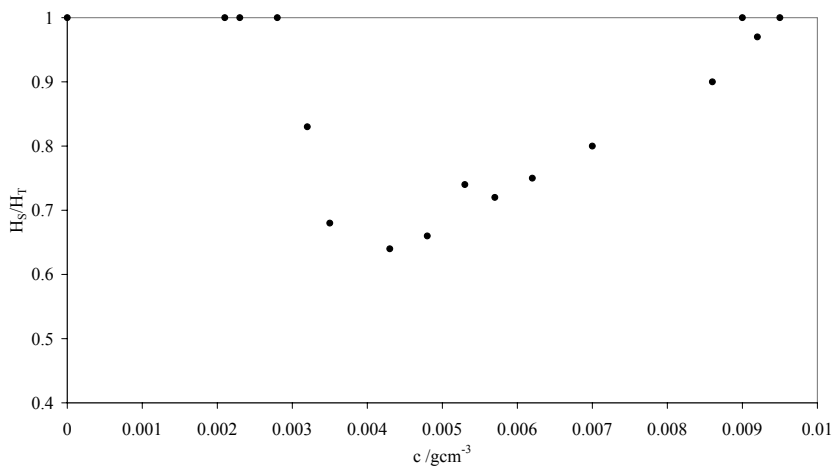

Figure 3

Typical phase separation results of PMMA with added PIB after $24 h\left(H K 8 a=180 \mathrm{~nm}, \delta=9 \mathrm{~nm}, \phi=0.113 M_{w}(P I B)=380000\right)$

380000 ) was different from that of $\mathrm{BaCO}_{3}$ suspensions.

Results showed that addition of high molecular mass nonadsorbing (free) polymer to particle dispersions effects a phase separation with a clear upper phase (supernatant) and particlerich (dense) phase. The phase separation was evident within a few hours with clear supernatant above some critical added polymer concentration (Goodwin et al., 2000). The formation of clear upper phases is often used as an indicator of flocculation that is due to depletion rather than bridging (Barrett, 1975). Progressive addition of the free polymer causes the colloid stability to be decreased markedly above a certain critical polymer, $c^{+}$. At lower polymer concentration, the dispersion remained a singlephase fluid with particles uniformly dispersed (stable) for relatively long periods. As the polymer concentration is increased to above $c^{+}$, phase separation takes place between particle-rich (dense) phase and solvent-rich (almost clear) top phase. Figures 2 and 3 show the plots of the ratio $H_{S} / H_{T}$ as a function of polymer concentration. Table 3 shows the observed $c^{+}$values at which the ratio $H_{S} / H_{T}$ begins to decrease. $c^{+}$decreases with increase in free polymer molecular mass and seemed to be independent of the adsorbed layer thickness. The dependence on particle fraction was minimal at low fractions used here $(\phi<0.35)$ although for similar systems, $\mathrm{c}^{+}$of the free polymer has been found to depend on its molecular mass and on the volume fraction of the suspension (Tadros, 1987). At higher polymer concentration, the ratio starts to rise due either due to increased viscosity of the continuous phase or simply a reduction in the extent of depletion or both. The viscosity of the medium increases with increase in polymer concentration. One way of correcting this is to multiply 
the ratio of heights by the ratio of the polymer solution viscosity to the solvent viscosity or simply relative viscosity $\left(\eta_{\mathrm{r}}\right)$ as follows:

$$
\frac{H_{S}}{H_{T}}(\text { corr })=\frac{H_{S}}{H_{T}} \eta_{r}
$$

This is effectively reducing the height of the phase boundary to the value it would have achieved had the viscosity of the solvent not been changed by the increasing polymer concentration. In fact this correction would also apply to the additional drag experienced by a particle during sedimentation. An example of the viscous correction is shown in Fig. 2.

\begin{tabular}{|l|c|c|c|}
\hline \multicolumn{4}{|c|}{ TABLE 3 } \\
$\begin{array}{c}\text { Summary of polymer-induced phase } \\
\text { separation critical polymer } \\
\text { concentrations }\end{array}$ \\
\hline Latex & Polymer & $\boldsymbol{\varphi}$ & $\mathbf{c}^{+} / \mathbf{g c m}^{-3}$ \\
\hline HK5 & P115K & 0.100 & 0.008 \\
HK5 & P31K & 0.311 & 0.023 \\
HK8 & PIB & 0.113 & 0.0028 \\
\hline
\end{tabular}

\section{Conclusions}

In this paper, it has been shown that the phase separation and stability behaviour of aqueous $\mathrm{BaCO}_{3}$ dispersions in the presence of polymers (xanthan gum, $\mathrm{CMC}$ and carrageenan) differs from that of non-aqueous PMMA dispersions with added nonadsorbing PIP. In the former, the phase separation and stability are mainly determined by the density differences between the particles and the continuous phase containing the polymer. Xanthan gum is a more effective dispersant and destabiliser of $\mathrm{BaCO}_{3}$ particles than $\mathrm{CMC}$ and carrageenan.

In the case of PMMA dispersions, the phase separation is due to depletion flocculation. There is a critical polymer concentration, $c^{+}$, above which phase separation occurs. The $c^{+}$values depend on polymer molecular mass.

\section{Acknowledgements}

Financial support from the Publications and Research Committees of the University of Botswana and the University of Namibia is gratefully acknowledged.

\section{References}

ANTL I, GOODWIN JW, HILL RD, OTTEWILL RH, OWENS SM, PAPWORTH S and WATERS JA (1986) The preparation of poly (methyl methacrylate) lattices in non-aqueous media. Coll. Surf. 17 $67-78$.

BARRETT KEJ (ed.) (1975) Dispersion Polymerisation in Organic Media $\left(1^{\text {st }}\right.$ edn.). Wiley, London.
DICKINSON E (1992) An Introduction to Food Colloids. Oxford University Press.

FRIEND JFC and LOEWENTHAL RE (1992) Stasoft III - Chemical Conditioning of Low and Medium Salinity Waters. Report No TT 53/92. Water Research Commission, Pretoria, RSA.

GOODWIN JW and HUGHES RW (2000) Rheology for Chemists. Royal Society of Chemistry, Cambridge.

GOODWIN JW, HUGHES RW, KWAAMBWA HM and REYNOLDS PA (2000) The phase separation behaviour and the viscoelastic properties of particles with non-adsorbing polymers: Part I - Experimental study. Coll. Surf A: Physicochem. Eng. Aspects 161 339-359.

GOODWIN JW, HUGHES RW, KWAAMBWA HM and REYNOLDS PA (2000) The phase separation behaviour and the viscoelastic properties of particles with non-adsorbing polymers: Part II - Theoretical study. Coll. Surf A: Physicochem. Eng. Aspects 161 361-376.

GOODWIN JW, HUGHES RW, KWAAMBWA HM and REYNOLDS PA (2004) Viscosity behaviour of particles with non-adsorbing polymers: Part III - Experimental study. Coll. Surf A: Physicochem. Eng. Aspects 233 163-170.

ISRAELACHVILI J (1992) Intermolecular and Surface Forces. Academic Press Ltd., London.

KOENDERINK GH, AARTS GALD, DE VILLENEUVE VWA, PHILLIPSE AP, TUINIER R and LEKKERKERKER HNW (2003) Morphology and kinetics of phase separating transparent xanthancolloid mixtures. Biomacrom. 4 129-136.

KWAAMBWA HM (1995) Rheological Studies of Concentrated Sterically Stabilized Colloidal Dispersions with Added Low Molecular Weight Polymers. Ph.D. Thesis, University of Bristol, Bristol.

LOEWENTHAL RE, WIECHERS HNS and MARAIS GvR (1986) Softening and Stabilisation of Municipal Waters. WRC Report No 24/86. Water Research Commission, Pretoria, RSA.

MSHINGENI KE and SEMESI AK (1977) Studies on carrageenans from economic red algal genus Eucheuma in Tanzania. Bot. Mar. 20 239-242.

NISHINI K and TAKAHASHI R (2003) Interaction in polysaccharide solutions and gels. Current Opinion in Colloid \& Interface Sci. 8 396-400.

NUSSINOVITCH A (1997) Hydrocolloids Applications: Gum Technology in the Food and Other Industries ( $1^{\text {st }}$ edn.) Blackie Academic \& Professional, London.

PARKER A, GUNNING PA, NG K and ROBINS MM (1995) Food Hydrocoll. 9 333-342.

POON CPC (1973) Electrochemical process for sewage treatment. Proc. $28^{\text {th }}$ Ind. Waste Conf. 281-291.

REYNOLDS PA and REID CA (1991) The effect of non-adsorbing polymers on the rheology of a concentrated non-aqueous dispersion. Langmuir 789.

TADROS THF (ed.) (1982) The Effect of Polymers on Dispersion Stability. Academic Press, New York/London.

TADROS THF (ed.) (1987) Solid/Liquid Dispersion. Academic Press, New York/London.

TRUSLAR GE, KERR CA, LOEWENTHAL RE and BUCKLEY CA (1991) The chemistry of sulphate removal processes utilizing barium carbonate. Proc. $2^{\text {nd }}$ WISA Bienn. Conf. Johannesburg, RSA.

VAN KRAVELEN DW (1976) Properties of Polymers: Their Estimation and Correlation with Chemical Structure. Elsevier Scientific, Amsterdam.

VERWEY EJW and OVERBEEK JTHG (1948) Theory of the Stability of Lyophobic Colloids. Elsevier, New York. 